\title{
Mean-field transport in stratified and/or rotating turbulence (Corrigendum)
}

\author{
A. Brandenburg ${ }^{1,2}$, K.-H. Rädler ${ }^{3}$, and K. Kemel ${ }^{1,2}$ \\ 1 NORDITA, AlbaNova University Center, Roslagstullsbacken 23, 10691 Stockholm, Sweden \\ e-mail: brandenb@nordita.org \\ 2 Department of Astronomy, AlbaNova University Center, Stockholm University, 10691 Stockholm, Sweden \\ 3 Astrophysical Institute Potsdam, An der Sternwarte 16, 14482 Potsdam, Germany
}

A\&A 539, A35 (2012), DOI: 10.1051/0004-6361/201117871

Key words. magnetohydrodynamics (MHD) - hydrodynamics - turbulence - Sun: dynamo - errata, addenda

An error occured during the production process. The top panel of Fig. 1 was truncated. The correct Fig. 1 is published on this page.

Fig. 1. The coefficients $\tilde{\alpha}_{\perp}, \tilde{\beta}_{\perp}, \tilde{\beta}_{\|}$, and $\tilde{\mu}$, as well as $\tilde{\beta}_{\perp}^{C}$ and $\tilde{\beta}_{\|}^{C}$ for the Roberts flow, calculated in the second-order correlation approximation, as functions of $k_{\perp} / k_{\mathrm{f}}$, where $k_{\mathrm{f}}=\sqrt{2} k_{0}$ is the effective wavenumber of the flow. Results obtained with $k_{x}=k_{y}$ and $k_{\|} / k_{\perp}=1 / \sqrt{2} \approx 0.7$ or $k_{\|} / k_{\perp}=1 / 16 \sqrt{2} \approx 0.004$ are represented by open squares and dotted lines or by open diamonds and dashed lines, respectively. Results with $k_{x} / k_{y}=0.75\left[\boldsymbol{k}_{\perp}=(3,4,0) k_{1}\right]$ or $k_{x} / k_{y}=5\left[\boldsymbol{k}_{\perp}=(5,1,0) k_{1}\right]$ and $k_{\|} / k_{\perp}=0.2$ are indicated by open or filled circles, respectively. Orange and black symbols correspond to the first and second expressions for $\tilde{\beta}_{\perp}$ and $\tilde{\mu}$ in (21) or for $\tilde{\beta}_{\perp}^{C}$ in (26).
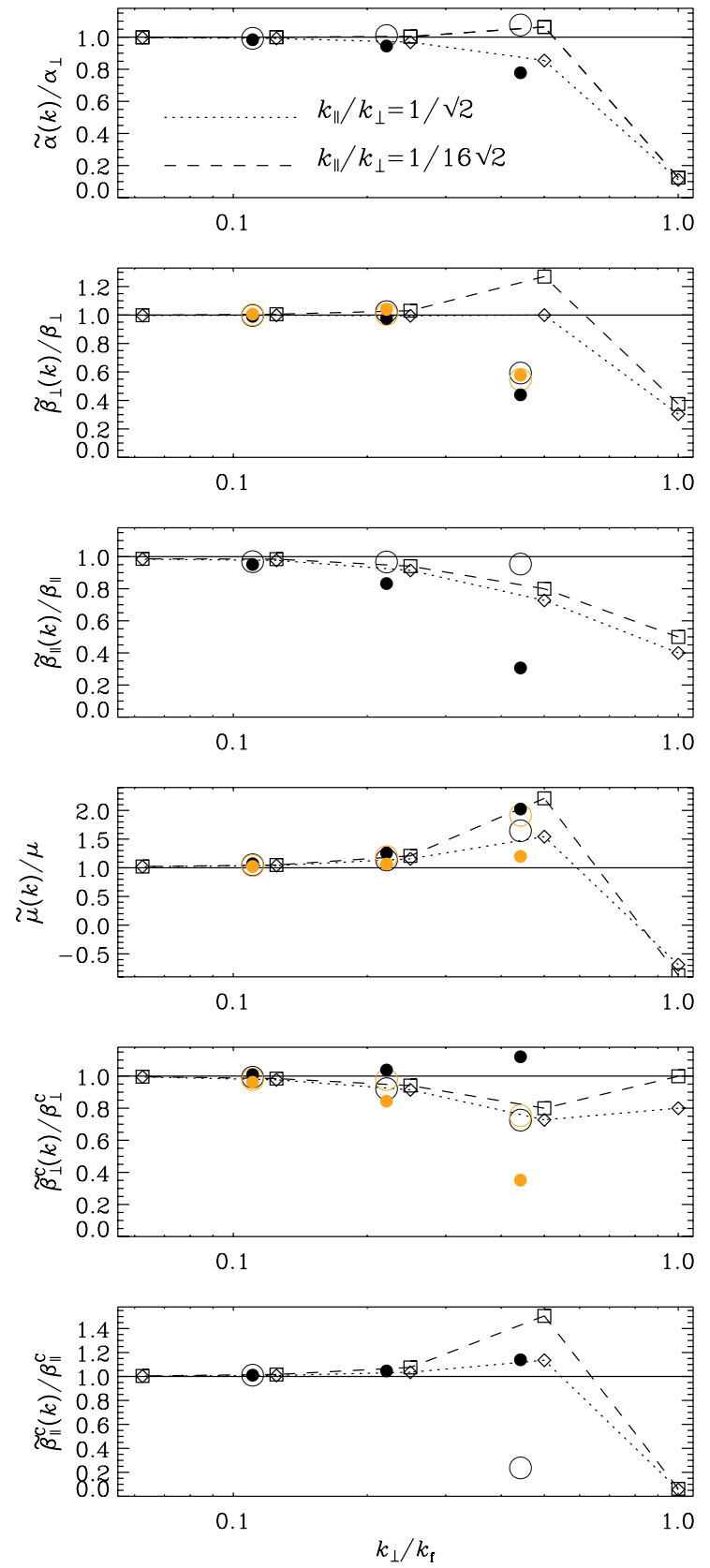

Article published by EDP Sciences 